\title{
Trastorno del Espectro Autista
}

Autistic Espectrum Disorder

\section{Trastorno del Espectro Autista: \\ La Historia}

\author{
Autistic Espectrum Disorder; The History \\ Dwight Denis Herrera-Del Aguila
}

\section{Resumen}

Son muchos los autores que han descrito el trastorno del espectro autista desde que las primeras referencias hechas por el monje Alemán Johannes Mathesius escritas en el siglo XVI. A pesar que en el siglo XX se acuñara el término “autismo" como parte de la descripción de la clínica del paciente con esquizofrenia hemos visto como a través del tiempo este concepto ha ido evolucionando hasta convertirse en una entidad diagnóstica propia descrita en los manuales de psiquiatría. Es por esto que el presente artículo se avocará en hacer un repaso de los principales autores que contribuyeron con la descripción del trastorno del espectro autista, así como su evolución en los manuales diagnósticos.

Palabras clave: Trastorno del espectro autista, autores.

\section{Abstract}

Many authors have described autism spectrum disorder since the first references made by the German monk Johannes Mathesius were written in the 16th century. Despite the fact that in the 20th century the term "autism" was coined as part of the description of the clinical picture of the patient with schizophrenia, we have seen how over time this concept has evolved to become its own diagnostic entity described in the manuals of psychiatry. This is why this article will focus on reviewing the main authors who contributed to the description of the autism spectrum disorder as well as its evolution in diagnostic manuals.

Keywords: Autism spectrum disorder, authors.

\section{Introducción}

Las primeras referencias que se hacen de pacientes con trastornos del espectro autista datan del siglo XVI. El monje alemán Johannes Mathesius reporta la historia de un muchacho de 12 años de características autísticas. Él dice que Martín Lutero, padre de la reforma protestante, describía al muchacho como una masa de carne sin espíritu implantado, que era poseído por el demonio cada cierto tiempo. Posteriormente en el siglo XVII, en el libro anónimo "Las florecillas de San
Francisco" se describe al Fraile Junípero Serra con diversas características de pacientes del trastorno del espectro autista tales como: Imposibilidad de entender las claves sociales y el lenguaje pragmático, dificultades para entender la intencionalidad del comportamiento de sus pares, dificultad para comprender el lenguaje no verbal ${ }^{(1)}$.

Fue Eugene Bleuler quien acuñó el término de autismo por primera vez en su obra "Dementia praecox oder Gruppe der Schizophrenien" para el tratado de psiquiatría realizado por

${ }^{1}$ Servicio de Psiquiatría, Hospital Nacional Guillermo Almenara Irigoyen, Lima - Perú. Departamento de Psiquiatría, Hospital Nacional Guillermo Almenara Irigoyen, Lima - Perú. Médico Residente de Psiquiatría. Universidad de San Martín de Porres. 
Gustav Aschaffenburg que se publicó en Viena en el año $1911^{(2)}$. El término hacía referencia a la pérdida del contacto con la realidad en el contexto de un paciente con esquizofrenia de edad adulta $\mathrm{o}$ adolescente $\mathrm{e}^{(3)}$.

Bleuler dilucidó un grupo de psicosis esquizofrénicas con características psicopatológicas en común, siendo la principal la Spaltung (escisión) que es la que da el nombre al grupo, además de otros síntomas importantes como el "autismo" o "autismos"(2). Bleuler utiliza un término proveniente del griego "autos" cuyo significado es "en sí mismo". Por tanto, la sintomatología autista estaría caracterizada por la pérdida de contacto con la realidad, la vivencia en su propio mundo fantástico y la incapacidad de establecer comunicación con sus pares ${ }^{(4)}$.

Unos años más tarde que el término fuera utilizado por Bleuler, Carl Jung acuñó los conceptos de introvertido y extrovertido para describir la personalidad de las personas. Carl Jung definía a las personas introvertidas como aquellas cuya atención estaba predominantemente dirigida a su mundo interno además de ser contemplativas. Se consideraba que la introversión en su grado más severo era lo que definía al autismo y esto como tal era característicos de algunos pacientes con esquizofrenia ${ }^{(1)}$.

Eugéne Minkowski, psiquiatra de origen francés y que trabajó con Bleuler en Burglözli, definió el término autismo como "la pérdida del contacto del 'Élan vital' con la realidad"(5). Destacó las conductas de dichos pacientes como disruptivas ${ }^{(6)}$.

Leo Kanner en 1943 introduce el término en el mundo infantil con el artículo "Autistic disturbances of affective

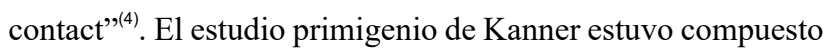
por una muestra de 11 niños que presentaban conductas extrañas ${ }^{(7)}$. Las características más saltantes de estos niños eran: incapacidad para establecer contacto con los demás, retraso y alteración en el uso del lenguaje y una obstinación pertinaz a mantener el ambiente sin cambios acompañada de realización de rituales ${ }^{(8)}$.

Hans Asperger, un pediatra austriaco, publicó en el año 1944 observaciones muy parecidas a las descritas por Kanner en los Estados Unidos. Asperger publicó la historia de 4 pacientes a los cuales describía bajo el término autista. Los caracterizó por falta de empatía, ingenuidad, dificultad para entablar amistades, lenguaje pedante y repetitivo, déficit en la comunicación no verbal, interés rígido por temas específicos, movimientos torpes y descoordinados. Estos pacientes además tenían la característica de hablar de forma detallada sobre sus temas de interés por lo que Asperger los llamó "kleine professoren", que en español significa "pequeños profesores ${ }^{\prime \prime(9)}$.

Cabe mencionar que, en el año de 1996, Sula Wolff, psiquiatra infantil de origen alemán, publicó la traducción de un artículo en ruso de la autora Grunya Sukhareva quien sería la primera en describir de forma detallada los síntomas autistas en niños en el año de $1925^{(10)}$.

\section{El autismo, el DSM y el CIE 10}

A pesar de que nueve años antes de la publicación del DSM I ya se había identificado al autismo como entidad específica, no fue incluido en esta clasificación. En el año 1962 cuando salió publicado el manual del DSM II tampoco apareció como una entidad como tal sino más bien como dependiente del espectro de la esquizofrenia infantil ${ }^{(1)}$.

Fue recién en el año 1980, con el nuevo manual DSM III, que se contempla al autismo como entidad diagnóstica propia $^{(11)}$ acuñándose el término "autismo infantil”. En el año 1987, cuando aparece el DSM III-R, se modifica el término "autismo infantil" por "Trastorno autista". Entre los años 1994 y 2000 aparecieron los manuales DSM IV y DSM IV TR, que definieron el autismo bajo 3 características primordiales: alteración en la interacción social, alteración en la comunicación, patrones de comportamiento, intereses, actividades restringidas y estereotipadas. En el DSM V se fortalece el concepto de autismo mediante la denominación: "Trastornos del espectro autista" que reemplaza al término "Trastornos generalizados del desarrollo". En esta nueva categoría se incluyen las siguientes entidades nosológicas: trastorno autista, trastorno de Asperger, trastorno desintegrativo infantil y trastorno generalizado del desarrollo no especificado. Dejándose de lado el trastorno de $\operatorname{Rett}^{(12)}$.

Por otro lado, la OMS en el año de 1974 incluyó en la Clasificación Internacional de Enfermedades, un capítulo en especial para los trastornos mentales, el capítulo $\mathrm{V}(\mathrm{F})$ a partir de la octava edición. El trastorno generalizado del desarrollo como se le conoció en el CIE 9 y CIE 10 ha pasado por importantes modificaciones hasta denominarse como trastorno del espectro autista en el CIE $11^{(2)}$. 
Referencias bibliográficas

1. Artigas-Pallares J, Paula I. El autismo 70 años después de Leo Kanner y Hans Asperger. Rev. Asoc. Esp. Neuropsiq. [Internet]. [citado 2020 Oct 03] 2012;32(115):567-587. Disponible en:http://scielo.isciii.es/scielo.php?script= sci_arttext\&pid=S0211-57352012000300008\&lng=es. http://dx.doi.org/10.4321/S0211-57352012000300008.

2. Garrabé de Lara J. El autismo: Historia y clasificaciones. Salud Ment [revista en la Internet]. [citado 2020 Oct 03] 2012;35( 3):257-261. Disponible en: http://www.scielo. org. $\mathrm{mx} / \mathrm{scielo}$.php? script=sci_arttext\&pid=S018533252012000300010\&lng=es.

3. Talero C, Martínez LE, Mercado M, Ovalle JP, Velásquez, A, Zarruk JG. Autismo: estado del arte. Revista Ciencias de la Salud [Internet]. 2003;1(1):6

4. Greydanus DE, Toledo-Pereyra LH. Historical perspectives on autism: its past record of discovery and its present state of solipsism, skepticism, and sorrowful suspicion. Pediatr Clin North Am. 2012 Feb;59(1):1-11, ix. doi: 10.1016/j.pcl.2011.10.0 04. PMID: 22284788.

5. Garrabé de Lara J. El autismo. Historia y clasificaciones. Salud Mental, 2012;35(3),257-261.[fecha de Consulta 30 de Mayo de 2021]. ISSN: 0185-3325. Disponible en: https://www.redalyc.org/articulo.oa? $\mathrm{id}=58223340010$

6. Reynoso C, Rangel MJ, Melgar V. El Trastorno del Espectro Autista: Aspectos etiológicos, diagnósticos y terapeúticos. Revista Médica del Instituto Mexicano del Seguro Social.
2017;214-222.

7. Balbuena Rivera F. Breve revisión histórica del autismo. Rev. Asoc. Esp. Neuropsiq. [Internet]. [citado 2020 Oct 03] 2007;27(2): 61-81. Disponible en: http://scielo.isciii.es/scielo. p h p ? s c r i p t = s c i a r t ex t \& p i d = S 0211 $57352007000200006 \& \operatorname{lng}=\mathrm{es}$.

8. López Gómez S, Rivas Torres R, Taboada Ares EM. Revisiones sobre el autismo. Revista Latinoamericana de Psicología. 2009:41.

9. Naranjo Flórez RA. (2014). Avances y perspectivas en Síndrome de Asperger. Nova, Retrieved 2021;12(21):81-101. from http://www.scielo.org.co/scielo.php?script=sci arttext\&pid=S1794-24702014000100007\&lng=en\&tlng=es.

10. Manouilenko I, Bejerot S. "Sukhareva: antes de Asperger y Kanner". Revista nórdica de psiquiatría (Reporte). 2015;69(6): 1761-1764.

11. Klin A. Autismo e síndrome de Asperger: uma visão geral. Rev. Bras. Psiquiatr. [Internet]. [cited 2020 Oct 03] 2006;28( Suppl 1):s3-s11. Available from: http://www.scielo.br/scielo.php? script=sci_arttext\&pid=S1516-44462006000500002\&lng=en. https://doi.org/10.1590/S1516-44462006000500002. doi:10.3109/08039488.2015.1005022. ISSN 1502-4725. PMID 25826582

12. Bonilla M, Chaskel R. Trastorno del espectro Autista. Curso contínuo de actualización en Pediatría, 2016;15,(1):19-29.

Contribución de autoría: Dwight Denis Herrera-Del Aguila ha participado en la concepción del artículo, la recolección de datos, su redacción y aprobación de la versión final. Conflicto de interés: El autor no tiene conflicto de interés con la publicación de este trabajo. Financiamiento: Autofinanciado.

Citar como: Herrera-Del Aguila DD. Trastorno del Espectro Autista. La Historia. Diagnóstico(Lima). 2021;60(3):131-133.

DOI: 10.33734 /diagnostico.v60i3.300

Correspondencia: Dwight Denis Herrera Del Aguila. Hospital Nacional Guillermo Almenara Irigoyen - Departamento de Psiquiatría. Av. Grau 800. Lima 1. Lima, Perú.

Correo electrónico: dwightherreradelaguila@gmail.com Teléfono: 982915968

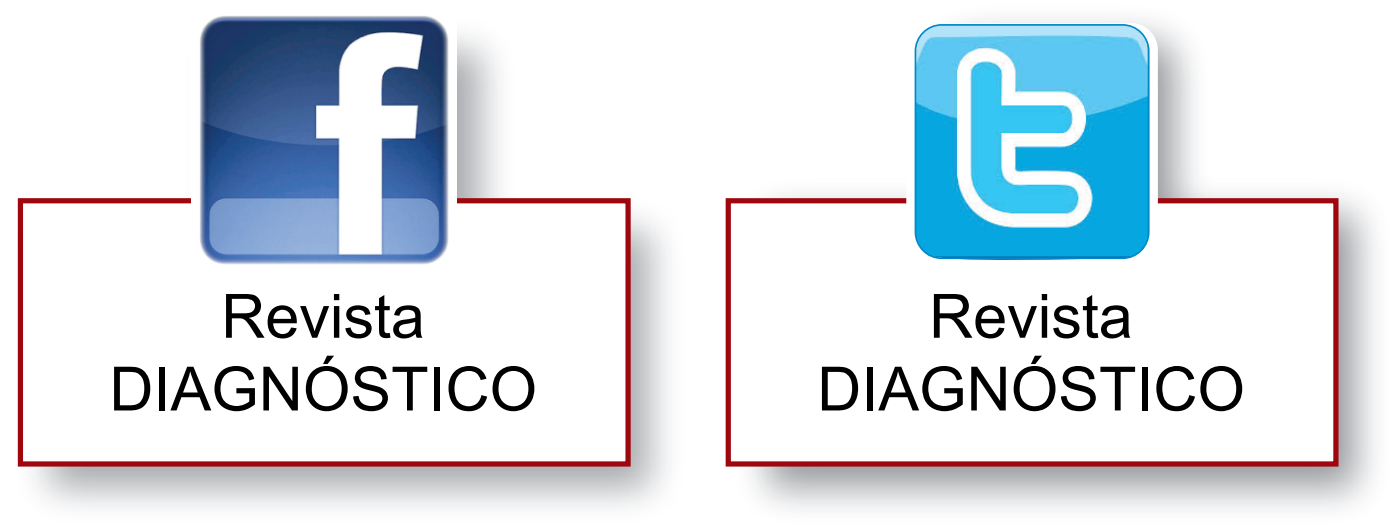

